\title{
Confined nucleation of calcium carbonate studied in the surface forces apparatus
}

\author{
J. DZIADKOWIEC ${ }^{1 *}$, B. ZAREEILPOLGARDANI ${ }^{2}$,
} D.K.DYSTHE ${ }^{1}$, A. RøYNE ${ }^{1}$

123

${ }^{11}$ NJORD Centre, University of Oslo, PObox 1048 Blindern, 0316 Oslo, Norway

${ }^{2}$ Institut Lumière Matière, Université Claude Bernard Lyon 1, campus de la Doua, 69622 Villeurbanne, France

joanna.dziadkowiec@fys.uio.no*

Amorphous, hydrated, and poorly crystalline calcium carbonate phases can be kinetically stabilized in spatial confinement. Do these species commonly exist within narrow spaces at reactive mineral contacts? What is their influence on mineral grain cohesion? In this work, we have explored the nucleation of $\mathrm{CaCO}_{3}$ between two macroscopic mineral surfaces using the surface forces apparatus. We show that even submicron-sized $\mathrm{CaCO}_{3}$ precipitate can exert a substantial force of crystallization on the solid, confining walls and generate long-range repulsion that prevents the surfaces from coming into adhesive contacts. The long-range repulsion and prolonged stabilization of the viscous, gel-like $\mathrm{CaCO}_{3}$ precipitate inside confined gaps can shape rock microstructures and affect their mechanical properties in a major way. 